\title{
reciamuc
}

Revista cientifica de investigación actualización del mundo de las ciencias

Nina Paulette Arteaga Vélez ${ }^{\text {a }}$, Ligia Elena Panezo Carbo ${ }^{\text {b; }}$ Andrea Geraldine

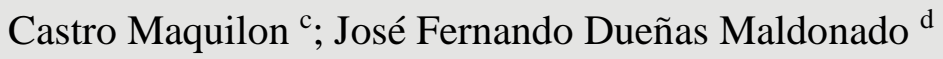

El bloqueo de nervios periféricos en el manejo del dolor postoperatorio

Peripheral nervous block as post-surgical pain control

Revista Científica de Investigación actualización del mundo de las Ciencias. Vol. 3 núm., 3, julio, ISSN: 2588-0748, 2018, pp. 863-880

DOI: $10.26820 /$ reciamuc/3.(3).julio.2019.863-880

URL: $\underline{\text { http://reciamuc.com/index.php/RECIAMUC/article/view/307 }}$

Código UNESCO: 2411.12 Fisiología del Sistema Nervioso Central

Tipo de Investigación: Artículo de Revisión

(C) RECIAMUC; Editorial Saberes del Conocimiento, 2019

Recibido: 28/04/2019

Aceptado: 19/05/2019

Publicado: 01/07/2019

Correspondencia: paulettearteaga@gmail.com
a. Médico; Investigador Independiente; Guayaquil, Ecuador; paulettearteaga@gmail.com
b. Médico Cirujano; Investigador Independiente; Guayaquil, Ecuador; queen_black85@ hotmail.com
c. Médico; Investigador Independiente; Guayaquil, Ecuador; andreacastroczs5@gmail.com
d. Médico; Investigador Independiente; Guayaquil, Ecuador; joseduenasmaldonado@gmail.com 


\section{EI bloqueo de nervios periféricos en el manejo del dolor postoperatorio}

Vol. 3, núm. 3., (2019)

Nina Paulette Arteaga Vélez; Ligia Elena Panezo Carbo; Andrea Geraldine Castro Maquilon; José Fernando Dueñas Maldonado

\section{RESUMEN}

Los bloqueos de nervios periféricos (BNP) se utilizan cada vez más como un componente de la analgesia multimodal y se puede administrar como una inyección única (sBNP) o infusión continua a través de un catéter perineural (cBNP). Se realizó una revisión cualitativa centrada en sBNP y cBNP con respecto a los beneficios, riesgos y oportunidades para optimizar la atención al paciente. Los meta análisis de ensayos controlados aleatorios han mostrado un control del dolor superiory reducciones en el consumo de opioides en pacientes que reciben BNP en comparación con los que reciben tratamiento intravenoso en una variedad de procedimientos quirúrgicos de extremidades superiores e inferiores. cBNP también se ha asociado con una reducción en el tiempo de descarga en comparación con sBNP. Riesgos de BNP, independientemente de técnica o ubicación de bloqueo, incluye punción vascular y sangrado, daño nervioso y toxicidad sistémica en anestesia local Las complicaciones específicas del sitio incluyen debilidad del cuádriceps en pacientes que reciben bloqueo del nervio femoral y punción pleural o bloqueo neuroaxial en pacientes que reciben bloqueo interescaleno. La mayor limitación de sBNP es la duración corta de la acción (12-24 horas). cBNP puede ser complicado por la obstrucción del catéter, la migración y la pérdida de anestesia local, así como la extracción accidental de catéteres.

Palabras Claves: Analgesia Multimodal; Bloqueo del Nervio Periférico; Dolor Postoperatorio. 
El bloqueo de nervios periféricos en el manejo del dolor postoperatorio

Vol. 3, núm. 3., (2019)

Nina Paulette Arteaga Vélez; Ligia Elena Panezo Carbo; Andrea Geraldine Castro Maquilon; José Fernando Dueñas Maldonado

\begin{abstract}
Peripheral nerve blocks (BNP) are increasingly used as a component of multimodal analgesia and can be administered as a single injection (sBNP) or continuous infusion through a perineural catheter (cBNP). A qualitative review focused on $\mathrm{sBNP}$ and $\mathrm{cBNP}$ was made regarding the benefits, risks and opportunities to optimize patient care. Meta-analyzes of randomized controlled trials have shown superior pain control and reductions in opioid use in patients receiving BNP compared to those receiving intravenous therapy in a variety of upper and lower extremity surgical procedures. cBNP has also been associated with a reduction in download time compared to sBNP. Risks of BNP, regardless of blocking technique or location, includes vascular puncture and bleeding, nerve damage and systemic toxicity in local anesthesia Specific site complications include weakness of the quadriceps in patients receiving femoral nerve block and pleural puncture or neuraxial block in patients receiving interscalene block. The greatest limitation of sBNP is the short duration of the action (12-24 hours). cBNP can be complicated by catheter obstruction, migration and loss of local anesthesia, as well as accidental catheter removal.
\end{abstract}

Key Words: Multimodal Analgesia; Peripheral Nerve Block; Postoperative Pain. 


\section{EI bloqueo de nervios periféricos en el manejo del dolor postoperatorio}

Vol. 3, núm. 3., (2019)

Nina Paulette Arteaga Vélez; Ligia Elena Panezo Carbo; Andrea Geraldine Castro Maquilon; José Fernando Dueñas Maldonado

\section{Introducción.}

La analgesia multimodal se refiere a la utilización de combinaciones de analgésicos de acción a través de diferentes mecanismos, y teniendo de este modo ventajas de aditivo o actividad sinérgica y reducir al mínimo los efectos adversos con dosis más grandes de un único analgésico (Joshi, Schug, \& Kehlet, 2014). Técnicas multimodales basadas en la evidencia son procedimientos específicos y puede incluir combinaciones de analgésicos sistémicos (por ejemplo, opioides, acetaminofeno, fármacos antiinflamatorios no esteroideos), analgesia neuroaxial (espinal, epidural, y la combinación espinal / epidural), infiltraciones locales y los bloqueos nerviosos periféricos (BNP).

Los beneficios de BNP son numerosos e incluyen mejorar la unificación de los resultados clínicos, económicos y humanísticos (Tabla 1). BNP se ha asociado con una mejoría en el control del dolor posoperativo y la reducción en el uso de opioides en una variedad de procedimientos quirúrgico (Chan, Fransen, Parker, Assam, \& Chua, 2014). La evitación de los opioides no sólo reduce al mínimo el riesgo de eventos adversos, pero también tiene importantes implicaciones para la salud pública, dado que los opiáceos prescritos al alta hospitalaria, que son a menudo en exceso de la cantidad necesaria para controlar el dolor postoperatorio, pueden servir como una fuente para el desvío. Otro beneficio de BNP incluye reducción en la utilización de recursos hospitalarios, la mejora de la recuperación postoperatoria, y la mejora en la satisfacción del paciente. 


\section{El bloqueo de nervios periféricos en el manejo del dolor postoperatorio}

Vol. 3, núm. 3., (2019)

Nina Paulette Arteaga Vélez; Ligia Elena Panezo Carbo; Andrea Geraldine Castro Maquilon; José Fernando Dueñas Maldonado

Teniendo en cuenta los muchos beneficios de BNP en la práctica, no es casualidad que su uso se ha extendido en las últimas décadas. BNP son ahora un componente común de la analgesia por tanto de la extremidad superior (por ejemplo, el bloqueo del plexo braquial usando interescalas, enfoques nerviosas supra o infraclaviculares, y axilares) y las extremidades inferiores (por ejemplo, el plexo lumbar, femoral, ciático, y bloquea ciático poplíteo, entre otros) (Stein, Srikumaran, Tan, Freehill, \& Wilckens, 2012). Los avances técnicos incluyen el uso de colocación de agujas guiada por ultrasonido y el movimiento de la utilización de una sola inyección de anestésico local (BNP de un solo tiro [SBNP]) a una infusión continua administrada usando un catéter perineural (continuo BNP [CBNP]). A medida que este uso se ha expandido tanto en el hospital como en el ámbito ambulatorio, se ha logrado una mayor comprensión de los riesgos potenciales de estos procedimientos y las necesidades no satisfechas.

\section{Tabla 1 Beneficios de BNP como un componente del régimen de analgesia postoperatoria} multimodal

- La mejora en el control del dolor postoperatorio y la reducción en el uso de opioides

- Reducción de la duración de la estancia hospitalaria

- Prevención de readmisiones hospitalarias

- Reducción de las náuseas y vómitos postoperatorios

- movimiento más rápido a la fase 2 de recuperación y / o de cuidados posanestésicos unidad de derivación

- A principios de la participación en la terapia física

- Mejora de la satisfacción del paciente 


\section{EI bloqueo de nervios periféricos en el manejo del dolor postoperatorio}

Vol. 3, núm. 3., (2019)

Nina Paulette Arteaga Vélez; Ligia Elena Panezo Carbo; Andrea Geraldine Castro Maquilon; José Fernando Dueñas Maldonado

El objetivo de este artículo es revisar la literatura reciente sobre SBNP y CBNP como un componente de la analgesia multimodal postoperativa, destacando beneficios, riesgos y oportunidades para optimizar la atención al paciente.

\section{Metodología.}

Para el desarrollo de este proceso investigativo, se plantea como metodología la encaminada hacia una orientación científica particular que se encuentra determinada por la necesidad de indagar en forma precisa y coherente una situación, en tal sentido (Davila, 2015) define la metodología "como aquellos pasos previos que son seleccionados por el investigador para lograr resultados favorables que le ayuden a plantear nuevas ideas”. (p.66)

Lo citado por el autor, lleva a entender que el desarrollo de la acción investigativa busca simplemente coordinar acciones enmarcadas en una revisión bibliográfica con el fin de complementar ideas previas relacionadas al bloqueo nervioso periférico como control de dolor postoperatorio a través de una revisión de literatura, para así finalmente elaborar un cuerpo de consideraciones generales que ayuden a ampliar el interés propuesto.

\section{Tipo de Investigación}

Dentro de toda práctica investigativa, se precisan acciones de carácter metodológico mediante las cuales, se logra conocer y proyectar los eventos posibles que la determinan, así como las características que hacen del acto científico un proceso interactivo ajustado a una realidad posible de ser interpretada. En este sentido, se puede decir, que la presente investigación corresponde al tipo documental, definido por Castro (2016), "se ocupa del estudio de problemas 


\section{El bloqueo de nervios periféricos en el manejo del dolor postoperatorio}

Vol. 3, núm. 3., (2019)

Nina Paulette Arteaga Vélez; Ligia Elena Panezo Carbo; Andrea Geraldine Castro Maquilon; José Fernando Dueñas Maldonado

planteados a nivel teórico, la información requerida para abordarlos se encuentra básicamente en materiales impresos, audiovisuales y /o electrónicos". (p.41).

En consideración a esta definición, la orientación metodológica permitió la oportunidad de cumplir con una serie de actividades inherentes a la revisión y lectura de diversos documentos donde se encontraron ideas explicitas relacionadas con los tópicos encargados de identificar a cada característica insertada en el estudio. Por lo tanto, se realizaron continuas interpretaciones con el claro propósito de revisar aquellas apreciaciones o investigaciones propuestas por diferentes investigadores relacionadas con el tema de interés, para luego dar la respectiva argumentación a los planteamientos, en función a las necesidades encontradas en la indagación.

\section{Fuentes Documentales}

El análisis correspondiente a las características que predomina en el tema seleccionado, llevan a incluir diferentes fuentes documentales encargadas de darle el respectivo apoyo y en ese sentido cumplir con la valoración de los hechos a fin de generar nuevos criterios que sirven de referencia a otros procesos investigativos. Para (CASTRO, 2016) las fuentes documentales incorporadas en la investigación documental o bibliográfica, "representa la suma de materiales sistemáticos que son revisados en forma rigurosa y profunda para llegar a un análisis del fenómeno". (p.41). Por lo tanto, se procedió a cumplir con la realización de una lectura previa determinada para encontrar aquellos aspectos estrechamente vinculados con el tema, con el fin de explicar mediante un desarrollo las respectivas apreciaciones generales de importancia. 


\section{El bloqueo de nervios periféricos en el manejo del dolor postoperatorio}

Vol. 3, núm. 3., (2019)

Nina Paulette Arteaga Vélez; Ligia Elena Panezo Carbo; Andrea Geraldine Castro Maquilon; José Fernando Dueñas Maldonado

\section{Técnicas para la Recolección de la Información}

La conducción de la investigación para ser realizada en función a las particularidades que determinan a los estudios documentales, tiene como fin el desarrollo de un conjunto de acciones encargadas de llevar a la selección de técnicas estrechamente vinculadas con las características del estudio. En tal sentido, (Bolívar, 2015), refiere, que es "una técnica particular para aportar ayuda a los procedimientos de selección de las ideas primarias y secundarias”. (p. 71).

Por ello, se procedió a la utilización del subrayado, resúmenes, fichaje, como parte básica para la revisión y selección de los documentos que presentan el contenido teórico. Es decir, que mediante la aplicación de estas técnicas se pudo llegar a recoger informaciones en cuanto a la revisión bibliográfica de los diversos elementos encargados de orientar el proceso de investigación. Tal como lo expresa, (Bolívar, 2015) "las técnicas documentales proporcionan las herramientas esenciales y determinantes para responder a los objetivos formulados y llegar a resultados efectivos" (p. 58). Es decir, para responder con eficiencia a las necesidades investigativas, se introdujeron como técnica de recolección el método inductivo, que hizo posible llevar a cabo una valoración de los hechos de forma particular para llegar a la explicación desde una visión general.

Asimismo, se emplearon las técnicas de análisis de información para la realización de la investigación que fue ejecutada bajo la dinámica de aplicar diversos elementos encargados de determinar el camino a recorrer por el estudio, según, (Bolívar, 2015) las técnicas de procesamiento de datos en los estudios documentales "son las encargadas de ofrecer al investigador la visión o pasos que debe cumplir durante su ejercicio, cada una de ellas debe estar 


\section{El bloqueo de nervios periféricos en el manejo del dolor postoperatorio}

Vol. 3, núm. 3., (2019)

Nina Paulette Arteaga Vélez; Ligia Elena Panezo Carbo; Andrea Geraldine Castro Maquilon; José Fernando Dueñas Maldonado

en correspondencia con el nivel a emplear" (p. 123). Esto indica, que para llevar a cabo el procesamiento de los datos obtenidos una vez aplicado las técnicas seleccionadas, tales como: fichas de resumen, textual, registros descriptivos entre otros, los mismos se deben ajustar al nivel que ha sido seleccionado.

\section{Resultados.}

Eficacia clínica del BNP

BNP vs opioides

La eficacia de SBNP para mejorar el control del dolor a corto plazo se ha demostrado en una serie de procedimientos quirúrgicos de las extremidades superior e inferior. En una revisión Cochrane de ensayos aleatorios en pacientes sometidos a cirugía mayor de rodilla, BNP utilizado en combinación con analgésicos sistémicos (principalmente opiáceos) fue asociado con puntuaciones significativamente más bajas de dolor en reposo de 0 a 72 horas después de la cirugía, pero no hay diferencia en el dolor en movimiento hasta 48 a 72 horas después de la operación, en comparación con los analgésicos sistémicos solos (Xu, Chen, Ma, \& Wang, 2014). Esta revisión incluyó una amplia gama de procedimientos quirúrgicos (TKA reparación, ligamento cruzado anterior [ACL], y meniscectomía), técnicas de bloqueo (SBNP y CBNP) y ubicaciones (femoral, femoral / ciático, canal aductor), muchos de los cuales se han investigado en más enfocadas revisiones sistemáticas. Un metaanálisis de ensayos aleatorizados comparando FNB de un solo disparo a los opioides analgésicos controlados por el paciente por vía intravenosa mostró una significativa reducción en el dolor en reposo y en movimiento durante un 


\section{EI bloqueo de nervios periféricos en el manejo del dolor postoperatorio}

Vol. 3, núm. 3., (2019)

Nina Paulette Arteaga Vélez; Ligia Elena Panezo Carbo; Andrea Geraldine Castro Maquilon; José Fernando Dueñas Maldonado

máximo de 24 y 48 horas, respectivamente, con significativo menor consumo de opioides durante un máximo de 48 horas(Chan, Fransen, Parker, Assam, \& Chua, 2014). Cuando continuos FNB se compararon con intravenosas controladas por el paciente, el dolor en reposo y el dolor en movimiento eran significativamente reducidas durante 48 y 72 horas, respectivamente. En un metaanálisis comparando el bloqueo del compartimiento del psoas única y continua a los opiáceos orales en pacientes sometidos a artroplastia total de cadera, puntuaciones de dolor en escala analógica visual fue significativamente reducida en pacientes que reciben cualquier tipo de bloqueo del compartimiento del psoas en hasta 24 horas postoperatorias.

El impacto de la BNP en la intensidad del dolor en pacientes sometidos a reparación del LCA no es tan clara. En una revisión Cochrane comparando BNP en combinación con analgesia sistémica a la analgesia sistémica sola ( $\mathrm{n}=3$ ensayos controlados aleatorios), intensidades de dolor en reposo y en movimiento no eran significativamente mejorado en pacientes sometidos a reparación del LCA al recibir BNP (Xu, Chen, Ma, \& Wang, 2014).

Las diferencias en los diseños de los estudios y los resultados han impedido en gran parte estudios de procedimientos quirúrgicos en extremidades superiores para ser combinados utilizando metaanálisis; Sin embargo, varios comentarios sistemáticos han proporcionado resúmenes cualitativos de la evidencia existente. En una revisión de los ensayos que comparan un solo disparo y bloqueo interescalénico continuo (ISB) a la inyección de solución salina o opioides para una cirugía de hombro, el control del dolor fue superior con ISB de disparo único para hasta 24 horas en 4 de 4 ensayos y con continua ISB durante hasta 48 horas en 2 de 2 


\section{El bloqueo de nervios periféricos en el manejo del dolor postoperatorio}

Vol. 3, núm. 3., (2019)

Nina Paulette Arteaga Vélez; Ligia Elena Panezo Carbo; Andrea Geraldine Castro Maquilon; José Fernando Dueñas Maldonado

intentos (Fredrickson MJ, 2010). Una opinión más que reciente que se centra en la cirugía artroscópica de hombro informó que todos los 10 estudios incluidos encontraron significativareducción en el dolor durante hasta 24 horas después de la cirugía, con reducciones en el uso de opioides en 8 de los 9 estudios que informaron este resultado (Hughes, Matava, Wright, Brophy, \& Smith, 2013).

$C B N P$ vs $S B N P$

La administración de anestésicos locales a través de infusión continua permite una duración de la analgesia significativamente larga que la de una sola inyección. En un análisis combinado de 21 estudios comparando CBNP a SBNP para la analgesia postoperatoria, escalas de dolor visuales analógicas y el dolor en reposo fueron significativamente menores en los pacientes que reciben CBNP en los días postoperatorios 0 , 1, y 2, pero no en el día. El consumo de opioides también se redujo significativamente en el grupo CBNP en los días 1 y 2.

La disponibilidad apropiada de CBNP ha permitido a pacientes para ser dados de alta con una bomba de infusión ambulatoria en lugar de permanecer en el hospital o recibir alternativas analgésicas (por ejemplo, opioides orales) en el hogar. En 3 diseños similares en pacientes sometidos a ATR, la artroplastia total de cadera, o artroplastia total de hombro, se encontró que la preparación para el alta hospitalaria, según lo medido por un adecuado analgésico, que no requieren opioides IV, y la capacidad de caminar al menos 30 m, se logró significativamente más rápido entre los pacientes que recibieron CBNP hasta el día 4 del postoperatorio en comparación con los que recibieron CBNP hasta la mañana después de la cirugía. 


\section{EI bloqueo de nervios periféricos en el manejo del dolor postoperatorio}

Vol. 3, núm. 3., (2019)

Nina Paulette Arteaga Vélez; Ligia Elena Panezo Carbo; Andrea Geraldine Castro Maquilon; José Fernando Dueñas Maldonado

Riesgos y limitaciones de BNP

Los riesgos potenciales de BNP, independientemente de la técnica o bloque catiónico, incluyen la punción vascular y sangrado, daño en los nervios, y la toxicidad sistémica de anestésico local (LAST). La colocación BNP usando la guía del ultrasonido se ha demostrado para reducir la incidencia de punción vascular (Abrahams, 2009). Las complicaciones neurológicas son de particular interés debido a que la duración de los síntomas puede extenderse por semanas o meses después de la cirugía. Estos eventos se describen típicamente por los pacientes como hormigueo, dolor a la presión, o alfileres y agujas, y están asociados con tanto SBNP y CBNP. La incidencia se ha reportado que ser tan alto como el 8,2\%, la evidencia encontrada sobre el riesgo relativo con SBNP vs CBNP. Un estudio mostró una mayor incidencia SBNP vs CBNP en pacientes que reciben bloques femorales.

Los signos y síntomas de la última son dependientes de la dosis y varían de sabor metálico, zumbido de oídos, y adormecimiento peribucal de embargo, paro cardiaco y muerte (Morau \& Ahern, 2010). Las limitaciones de lugar de aplicación para el BNP incluyen debilidad de los cuádriceps en pacientes que reciben FNB, lo que puede aumentar el riesgo de caídas, aunque esto es controversial. Los estudios retrospectivos han encontrado un mayor riesgo de caídas en pacientes sometidos a ATR con CHN. Sin embargo, en 3 análisis combinado de ensayos controlados con placebo, los pacientes con menor CBNP con ropivacaína tenían significativamente más caídas de los pacientes que recibieron solución salina perineural (7\% frente al 0\%; $\mathrm{P}=0,013)$. Sin embargo, los autores señalan que evitar el uso de CBNP es poco 


\section{El bloqueo de nervios periféricos en el manejo del dolor postoperatorio}

Vol. 3, núm. 3., (2019)

Nina Paulette Arteaga Vélez; Ligia Elena Panezo Carbo; Andrea Geraldine Castro Maquilon; José Fernando Dueñas Maldonado

probable que elimine el riesgo de caídas y pueden tener un impacto negativo sobre el manejo del dolor.

Los BNP en extremidades superiores se pueden complicar por punción pleural y colocación de la aguja neuro axial centro (Fredrickson MJ, 2010). Neumotóraxha sido informado recientemente en $0,2 \%$ de los pacientes que han recibido continúa ISB continua. En una revisión sistemática de 13 estudios prospectivos y retrospectivos en pacientes que reciben OSI, (Moore, Maerz, \& Anderson, 2013) informaron que los efectos adversos que incluyen disnea, ronquera, síndrome de Horner, y el bloque no se produjeron en el 8,14\% de los pacientes. Sin embargo, un análisis retrospectivo de 17.157 pacientes sometidos a artroplastia total de hombrono encontró ningún aumento en el riesgo de complicaciones pulmonares o necesidad de ventilación mecánica entre los pacientes que recibieron ISB en combinación con anestesia general comparado con anestesia general sola.

BNP de un solo tiro

La principal limitación de SBNP es la corta duración de la acción de la mayoría de los anestésicos locales. Como tal, SBNP es el más adecuado para los procedimientos de cirugia en la que no se espera que el dolor postoperatorio exceda las 12-24 horas de duración; de lo contrario, los pacientes están en riesgo de dolor de rebote después de la descarga. La administración de volúmenes más grandes o mayores concentraciones de anestésicos locales puede aumentar la duración del bloqueo, sino también aumentar el riesgo de bloqueo motor y LAST. Por lo tanto, los métodos alternativos de superar estas limitaciones para procedimientos quirúrgicos con dolor que persiste más allá del primer día del postoperatorio son requeridos. 


\section{EI bloqueo de nervios periféricos en el manejo del dolor postoperatorio}

Vol. 3, núm. 3., (2019)

Nina Paulette Arteaga Vélez; Ligia Elena Panezo Carbo; Andrea Geraldine Castro Maquilon; José Fernando Dueñas Maldonado

\section{BNP continúo}

La incidencia de complicaciones CBNP depende de la localización, técnica de inserción y el bloque y por lo tanto, es dificultoso hacer generalizaciones entre los estudios. Menores complicaciones incluyen desalojamiento del catéter, obstrucción y fuga en el sitio del catéter. Las tasas de desplazamiento del catéter en estudios de voluntarios que participan en actividades de la vida diaria fueron tan altas como 25\%. (Marhofer \& Marhofer, 2013). Aunque las tasas de colonización bacteriana en el cateter aparecen alta, una infección clínicamente relevante es rara. Los factores de riesgo para la colonización entre los pacientes que recibieron la inserción del catéter guiada por ultrasonido incluyen duración del catéter en 48 horas, la diabetes, y la administración de antibióticos durante el mes previo a la cirugía. Por el contrario, una sola inyección de BNP a cabo bajo la guía del ultrasonido no se ha asociado con la infección.

Los costos asociados con CBNP son una importante consideración de los proveedores y hospitales que aplican esta técnica. Estos costes incluyen bombas de infusión, catéteres y otras subcapas; anestésicos locales; y proveedor tiempo requerido para la educación del paciente y el seguimiento. Los sitios que utilizan CBNP pueden volver a alize ahorro de costes con el uso de reutilizable vs bombas desechables y usando bomba basal de tasa fijo en comparación con bombas de bolo con tasa variable. CBNP ciático guiado por ultrasonido se ha demostrado ser más rentable que la estimulación del nervio para la colocación del catéter, con un pliegue en los costes de equipo que está siendo compensado por una reducción en el tiempo de enfermería postoperatorio. 


\section{El bloqueo de nervios periféricos en el manejo del dolor postoperatorio}

Vol. 3, núm. 3., (2019)

Nina Paulette Arteaga Vélez; Ligia Elena Panezo Carbo; Andrea Geraldine Castro Maquilon; José Fernando Dueñas Maldonado

Ya sea que CBNP se utilice en un paciente hospitalizado o ambulatorio, los recursos necesarios para proporcionar esta terapia segura y efectiva son sustanciales. Instalaciones de aplicación de CBNP estan obligadas primero a invertir en el desarrollo de la infraestructura apropiada (políticas y protocolos, los canales de comunicación) y luego comprometer recursos para la educación del paciente y el proveedor y seguimiento para garantizar los mejores resultados posibles para los pacientes. Consideraciones prácticas para el uso doméstico de CBNP incluyen la selección apropiada del paciente, el seguimiento y la educación sobre el manejo de la bomba y la eliminación. Los pacientes para quienes la CBNP ambulatorios puede ser inapropiados incluyen aquellos con insuficiencia renal y hepática, enfermedad pulmonar o de corazón (entre los pacientes con OSI), problemas de estado mental o alterados de manera sicosocial, incapacidad para ponerse en contacto después de la descarga o para acceder a un centro médico en caso de emergencia y falta de voluntad para aceptar la responsabilidad de la gestión de la bomba. Antes de la descarga, los pacientes deben ser educados en el cuidado del sitio del catéter y vestidor, cuándo parar la bomba en busca de signos de toxicidad, de cómo solucionar cualquier problema catéter o la bomba, cuándo llamar al médico o enfermera al notar signos de infección o problemas con el sistema de infusión, e instrucciones para la extracción del catéter.

Protocolos CBNP ambulatorios difieren en la frecuencia y modo de contacto con los pacientes después del alta, que van desde instrucciones escritas sólo para las consultas de enfermería en casa y la estrategia apropiada debe ser determinada sobre una base caso por caso con consideración para el tipo de cirugía y características del paciente. A pesar de seguimiento previsto, sin embargo, se requiere un proveedor de cuidado de la salud que esté disponible 24 


\section{EI bloqueo de nervios periféricos en el manejo del dolor postoperatorio}

Vol. 3, núm. 3., (2019)

Nina Paulette Arteaga Vélez; Ligia Elena Panezo Carbo; Andrea Geraldine Castro Maquilon; José Fernando Dueñas Maldonado

horas al día, 7 días a la semana para abordar las preocupaciones y preguntas de los pacientes. la retirada del catéter por lo general puede ser realizada por el paciente o un cuidador.

\section{Oportunidades}

La técnica ideal BNP tendría una duración de acción suficiente para proporcionar alivio del dolor durante el período más intenso del dolor postoperatorio, pero no resulta en un bloque de motor denso que podría ser desagradable para el paciente o dar lugar a problemas de seguridad, tales como caídas. Por otra parte, el riesgo de infección, de complicaciones neurológicas y sangrado debe reducirse al mínimo en la medida posible. La técnica debe ser fácil de realizar y por lo tanto depende en la habilidad técnica del anestesiólogo y con la mínima posibilidad de procedimientos fallidos. Por último, la técnica ideal BNP debe ser conveniente para los pacientes y fácil de manejar en el postoperatorio.

Actualmente las técnicas disponibles BNP están a la altura de este ideal en un número de maneras. SBNP es fácil de realizar, evita las preocupaciones asociadas con catéteres permanentes CBNP, y no requiere que el paciente sea responsable de la medicación y administración en casa, pero la duración del bloqueo es a menudo insuficiente para controlar el dolor más allá del primer día del postoperatorio. CBNP tiene las ventajas de una duración prolongada de la analgesia al administrar soluciones más diluidas de anestésico local (y por lo tanto minimizando el riesgo de LAST). Sin embargo, las tasas de desalojamiento del catéter pueden ser inaceptable, no todos los pacientes están dispuestos a aceptar la responsabilidad de CBNP casa, y la educación extensa y seguimiento son necesarios para la utilización con éxito. 


\section{El bloqueo de nervios periféricos en el manejo del dolor postoperatorio}

Vol. 3, núm. 3., (2019)

Nina Paulette Arteaga Vélez; Ligia Elena Panezo Carbo; Andrea Geraldine Castro Maquilon; José Fernando Dueñas Maldonado

\section{Conclusiones.}

Las técnicas de bloqueo de nervios periféricos están ahora comúnmente incorporados dentro de las estrategias de analgésicos postoperatorios multimodales. Las consecuencias de uso ampliado de BNP incluyen la mejora en el alivio del dolor y la necesidad de opiáceos postoperatorias, además de la mejora de la recuperación postoperatoria y menos eventos adverso relacionados con los opioides. Como una extensión de estos beneficios, los pacientes pueden ser dados de alta del hospital antes, y los procedimientos quirúrgicos son capaces de llevar a cabo en régimen ambulatorio.

A pesar de estos avances, hay espacio para la mejora en la prestación de tratamiento del dolor postoperatorio. Aunque CBNP se dirigió a la principal limitación de SBNP, se ha introducido un nuevo conjunto de dificultades en la técnica, las necesidades de educación del paciente y las complicaciones. Para los pacientes cuidadosamente seleccionados y anestesiólogos bien entrenados, CBNP puede ser una estrategia de tratamiento del dolor postoperatorio seguro y efectivo. Desafortunadamente, la mayor complejidad asociada con un catéter permanente y bomba aumenta la probabilidad de fracaso de la técnica (es decir, el desalojamiento del catéter, dobleces o fugas), y hay muchos pacientes en los que CBNP no es apropiado debido a a condiciones comórbidas y problemas logísticos.

\section{Bibliografía.}

Abrahams, M. (2009). Ultrasound guidance compared with electrical neurostimulation for peripheral nerve block. Anaesthesia, 408-417.

Bolívar, J. (2015). Investigación Documental. México. Pax. 


\section{EI bloqueo de nervios periféricos en el manejo del dolor postoperatorio}

Vol. 3, núm. 3., (2019)

Nina Paulette Arteaga Vélez; Ligia Elena Panezo Carbo; Andrea Geraldine Castro Maquilon;

José Fernando Dueñas Maldonado

Castro, J. (2016). Técnicas Documentales. México. Limusa.

Chan, E., Fransen, M., Parker, D., Assam, P., \& Chua, N. (2014). Femoral nerve blocks for acute postoperative pain after knee replacement surgery. Cochrane Database Syst Rev.

Davila, A. (2015). Concepto de terminos cientificos. Caracas: Oasis.

Fredrickson MJ, K. S. (2010). Postoperative analgesia for shoulder surgery: a critical appraisal and review of current techniques. Aneasthesia, 608-624.

Hughes, M., Matava, M., Wright, R., Brophy, R., \& Smith, M. (2013). Interscalene brachial plexus block for arthroscopic shoulder surgery. J Bone Joint Surg Am, 1318-1324.

Joshi, G., Schug, S., \& Kehlet, H. (2014). Procedure-specific pain management and outcome strategies. Best Pract Res Clin Anaesthesiol , 191-201.

Marhofer, D., \& Marhofer, P. (2013). Dislocation rates of perineural catheters. Anaesthesia, 800806.

Moore, D., Maerz, T., \& Anderson, K. (2013). Shoulder surgeons' perceptions of interscalene nerve blocks and a review of complications rates in the literature. Phys Sportsmed, 7784.

Morau, D., \& Ahern, S. (2010). Management of local anesthetic toxicity. Int Anesthesiol, 117.

Stein, B., Srikumaran, U., Tan, E., Freehill, M., \& Wilckens, J. (2012). Lowerextremity peripheral nerve blocks in the perioperative pain management of orthopaedic patients: AAOS exhibit selection. J Bone Joint Surg Am, 167.

Xu, J., Chen, X., Ma, C., \& Wang, X. (2014). Peripheral nerve blocks for postoperative pain aftermajor knee surgery. Cochrane Database Syst Rev.

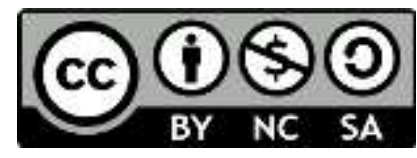

RECONOCIMIENTO-NOCOMERCIAL-COMPARTIRIGUAL

CC BY-NC-SA

ESTA LICENCIA PERMITE A OTROS ENTREMEZCLAR, AJUSTAR Y CONSTRUIR A PARTIR DE SU OBRA CON FINES NO

COMERCIALES, SIEMPRE Y CUANDO LE RECONOZCAN LA AUTORÍA Y SUS NUEVAS CREACIONES ESTÉN BAJO UNA LICENCIA CON LOS MISMOS TÉRMINOS. 